medium spiny neurons in adult but not in adolescent rats susceptible to diet-induced obesity. Psychopharmacology 233: 773-784.

Robinson MJ, Burghardt PR, Patterson CM, Nobile CW, Akil H, Watson SJ et al (2015). Individual differences in cue-induced motivation and striatal systems in rats susceptible to diet-induced obesity. Neuropsychopharmacology 40: 2113-2123.

Stice E, Figlewicz DP, Gosnell BA, Levine AS, Pratt WE (2013). The contribution of brain reward circuits to the obesity epidemic. Neurosci Biobehav Rev 37(9 Pt A): 2047-2058.

Tang DW, Fellows LK, Small DM, Dagher A (2012). Food and drug cues activate similar brain regions: a meta-analysis of functional MRI studies. Physiol Behav 106: 317-324.

Wolf ME (2016). Synaptic mechanisms underlying persistent cocaine craving. Nat Rev Neurosci 17: 351-365.

Neuropsychopharmacology Reviews (2017) 42, 36I-362; doi: 10.1038/npp.2016.221

\section{The Emerging Role of Innate Immunity in Alzheimer's Disease}

The amyloid cascade hypothesis is the dominant model for Alzheimer's disease $(\mathrm{AD})$ pathology and has guided $\mathrm{AD}$ drug development strategies for over two decades. The hypothesis states that excessive cerebral accumulation of $\beta$-amyloid plaques from oligomerization of amyloid- $\beta$ protein $(\mathrm{A} \beta)$ drives a neurodegenerative cascade, including generation of neurofibrillary tangles (NFTs) from tau protein. NFTs are a hallmark pathology for $\mathrm{AD}$. Overwhelming genetic and biochemical data support the primacy of $\mathrm{A} \beta$ fibrillization pathways in $\mathrm{AD}$ pathology. A landmark study by Choi et al (2014) also recently confirmed that $\beta$-amyloid generation leads to NFTs in novel 3D human neuronal cell culture models that recapitulate $\mathrm{AD}$ pathologies (Choi et al, 2014). However, the roles played by $\mathrm{A} \beta$ and $\beta$-amyloid in the amyloid cascade model has shifted markedly during the last two decades. Initially, insoluble $\beta$-amyloid plaques were viewed as driving neurodegeneration. The key species mediating $\mathrm{AD}$ pathology are now thought to be soluble oligomeric intermediates generated during $\mathrm{A} \beta$ fibrillization. The near future may see

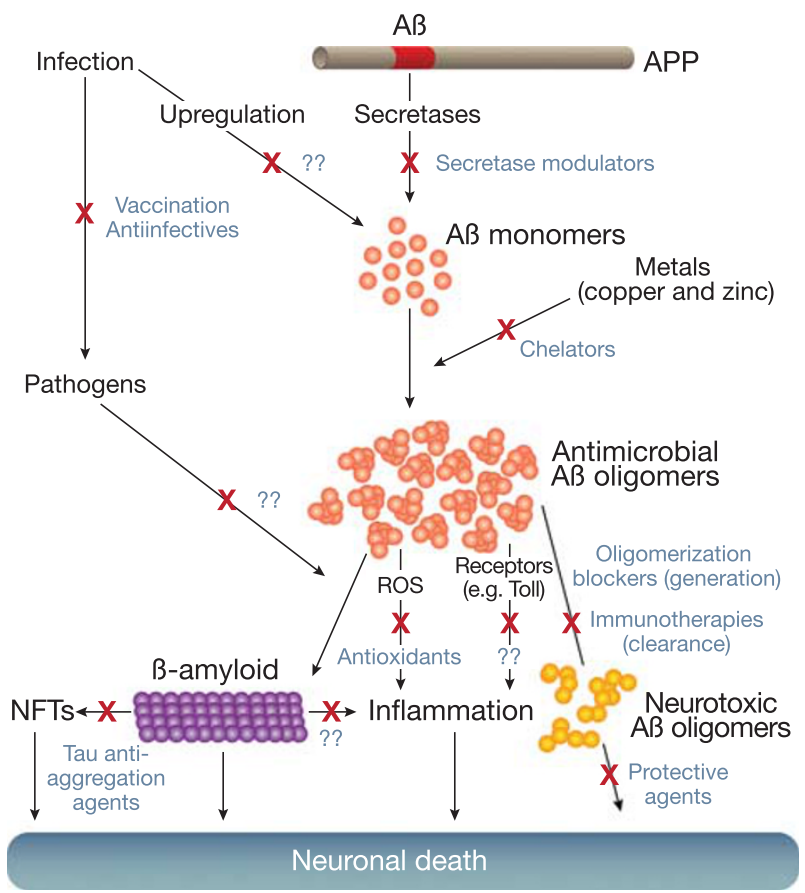

Figure 1. A $\beta$ oligomer pathways and potential targets for therapeutic intervention. Recent studies of 'resilient' brains with high senile plaque loads have shown $\beta$-amyloid alone is insufficient for AD neurodegeneration. Neurofibrillary Tangles (NFTs) and neuroinflammation are also required. Shown are oligomer-mediated pathways contributing to neurodegeneration in $A D$ and potential points for intervention $(\mathbf{X})$. Possible approaches emerging from the antimicrobial peptide role of $A \beta$ are indicated (??) as well as established therapeutic strategies, including secretase modulators that reduce the 42 amino acid A $\beta$ isoforms (eg, GSM, NS-GSI, BSI), copper chelators (eg, PBT2), A $\beta$ oligomerization blockers (eg, PBT2, ELND005, NRM8499), immunotherapies aimed at antibodymediated clearance of $\mathrm{A} \beta$ (eg, Bapi, Sola), antioxidants that attenuate ROS-mediated inflammation (eg, vitamin E, epigallo-catechine gallate, resveratrol), agents purported to be neuroprotective (eg, dimebon), and drugs that inhibit tau aggregation and NTF generation (eg, rhodanine-based and Phenylthiazolyl-hydrazide inhibitors).

further substantial adjustments to the amyloid cascade model if recent findings of an antimicrobial function for $\mathrm{A} \beta$, and links between $\mathrm{AD}$ and innate immune genes (Bertram and Tanzi, 2012) are confirmed. Importantly, new innate immune models of $\mathrm{AD}$ amyloidosis are likely to be of considerable interest for ongoing and future $\mathrm{AD}$ therapeutic efforts (Figure 1).

In prevailing models of $\mathrm{AD}$ amyloidogenesis, $\mathrm{A} \beta$ 's propensity to oligomerize and generate $\beta$-amyloid is viewed as an intrinsically abnormal and exclusively pathological activity. However, recent findings suggest $\mathrm{A} \beta$ oligomerization may help protect the brain from infection. $\mathrm{A} \beta$ shows potent in vitro microbicidal activities against human pathogens, consistent with identity as an antimicrobial peptide (Soscia et al, 2010). A $\beta$ expression also significantly increases survival of transgenic mice in acute encephalitis models (Kumar et al, 2016). Moreover, fibrillization appears to mediate the protective activities of $\mathrm{A} \beta$ in brain and leads to the concentration and entrapment of pathogens within $\beta$-amyloid deposits (Kumar et al., 2016). Confirmation of a physiological role for $\beta$-amyloid generation would shift the modality of $\mathrm{A} \beta$ 's pathophysiology from abnormal stochastic behavior toward dysregulated innate immune response. This suggests a response to perceived immunochallenge may mediate $\mathrm{AD}$ amyloidosis. This may involve sterile inflammatory stimuli, as with aseptic inflammation diseases. Or alternatively, $\mathrm{AD}$ amyloidosis may be driven by subclinical chronic cerebral infection. It remains unclear if infection precedes the establishment of the amyloid cascade in $\mathrm{AD}$ brain. 
However, a large body of data has accrued linking brain pathogens to enhanced

$\beta$-amyloid deposition (Miklossy, 2011). If an $\mathrm{AD}$ infection etiology is confirmed, patients could be given antiinfectives to slow or halt the diseases progression. Vaccination against ADlinked pathogens may also provide long-term protection. In any case, independent of the involvement of infection in AD etiology, the identification of an antimicrobial function for $\mathrm{A} \beta$ and emerging roles for innate immune pathways in $\beta$-amyloid generation seem poised to take the amyloid cascade hypothesis in new directions. Most importantly, these new findings seem likely to reveal much needed new therapeutic approaches for what has proven a highly intractable disease.

\section{FUNDING AND DISCLOSURE}

Our work is supported by grants from NIH (5R01AI081990-02), the Cure Alzheimer's Fund, and The Helmsley Charitable Trust. RDM. is a coinventor on a patent for the use of amyloidogenic peptides such as $\mathrm{A} \beta$ as possible clinical antibiotics. The remaining authors declare no conflict of interest.

\section{Deepak Kumar Vijaya Kumar ${ }^{1}$ and Robert D Moir ${ }^{1}$ \\ ${ }^{1}$ Genetics and Aging Research Unit, Department of Neurology, Massachusetts General Hospital and Harvard Medical School, Boston, MA, USA E-mail: moir@helix.mgh.harvard.edu}

Bertram L, Tanzi RE (2012). The genetics of Alzheimer's disease. Prog Mol Biol Transl Sci 107: 79-100.

Choi SH, Kim YH, Hebisch M, Sliwinski C, Lee S, D'Avanzo C et al (2014). A three-dimensional human neural cell culture model of Alzheimer's disease. Nature 515: 274-278.

Kumar DK, Choi SH, Washicosky KJ, Eimer WA, Tucker S, Ghofrani J et al (2016). Amyloid- $\beta$ peptide protects against microbial infection in mouse and worm models of Alzheimer's disease. Sci Trans/ Med 8: 340ra-372.

Miklossy $J$ (2011). Emerging roles of pathogens in Alzheimer disease. Expert Rev $\mathrm{Mol} \mathrm{Med}$ 13: e30.

Soscia SJ, Kirby JE, Washicosky KJ, Tucker SM, Ingelsson M, Hyman B et al (2010). The Alzheimer's disease-associated amyloid $\beta$-protein is an antimicrobial peptide. PLoS One 5: e9505.

Neuropsychopharmacology Reviews (2017) 42, 362-363; doi: 10.1038/npp.2016.226

\section{Pramipexole in Treatment Resistant- Depression, Possible Role of Inflammatory Cytokines}

Anhedonia is one of the most important predictors to developing treatment-resistant depression. The 'interest-activity' symptom dimension that includes loss of interest, diminished activity, and inability to make decisions has been shown to predict poor outcome of antidepressant treatment in large prospective clinical studies (Uher et al, 2012). Anhedonia symptoms can be induced experimentally in animals and humans by inflammatory cytokines, including interferon-alpha. Cytokines can affect dopamine function in the basal ganglia. Associations between altered dopamine function and impaired cortical-striatal reward circuitry are found in patients with major depression who display increased peripheral inflammatory markers and cytokines that include IL-6, TNF-alpha, and CRP (Felger and Miller, 2012). Anhedonia is not unique to depression. As a transdiagnostic psychopathological domain that appears in various psychiatric and medical conditions, anhedonia may receive pathogenic contributions from common cellular immunity mechanisms that affect reward systems (Swardfager et al, 2016). SSRIs and other first-line antidepressants fail to alleviate IFN-induced anxiety and depressive symptoms. Traditional stimulants that increase dopamine release and methylphenidate that blocks its reuptake have minimal effects on fatigue and anhedonia in depressed patients with inflammation-associated medical conditions such as advanced cancer. These findings suggest potential roles for cellular inflammation in mediating the development of treatment resistance to traditional antidepressants and stimulants, specifically when fatigue and anhedonia persist. In Parkinson's disease, where depression is common and anhedonia is a prominent feature, L-Dopa and other non-receptor specific dopamine agonists display little efficacy in preventing

or treating depression. However, pramipexole, a relatively selective D3 dopamine agonist has shown to relieve depression in Parkinson's disease. Also, in chronic and severe treatment-resistant depressed patients, including bipolar disorder, pramipexole at high doses has shown promising response (Fawcett et al, 2016). The selective expression of D3 receptors in the mesolimbic projection areas including the nucleus accumbens makes this dopamine receptor a promising target to overcome treatment-resistant depression where anhedonia symptoms may be perpetuated by inflammatory cytokines, such as in severe medical conditions with known increased levels of inflammation. The effects of pramipexole on brain immunological mechanisms are not fully understood. However, recent data suggest potentially important roles. Pramipexole attenuates the development of experimental autoimmune encephalomyelitis in mice, an animal model for multiple sclerosis (Lieberknecht et al, 2016). D3 receptors can be found in CD4-positive $\mathrm{T}$ cells, which are involved in the modulation of peripheral immune responses and promote neuro-inflammation in a murine model of Parkinson's disease (Contreras et al, 2016). Future studies in treatment-resistant depression that use D3-preferring and other dopamine agonists should monitor inflammatory markers as well as specific measures of anhedonia to better understand the role of inflammation in anhedonia and treatment resistance.

\section{FUNDING AND DISCLOSURE}

This article used no funding. Dr Escalona has no disclosures to make. Dr Fawcett has received speaking fees from the American Psychiatric Association and the Nevada Psychiatric Association and serves on a data review board for Amgen, and he has received remuneration as co-principal investigator of an NIMH-funded study. 